\title{
Chiral Symmetry Breaking Induced by Temperature Fluctuation in an Open System to Mimic Chirality Evolution in Nature
}

\author{
Weichun Pan ${ }^{1, a^{*}}$ Jin $\mathrm{Xu}^{2, \mathrm{~b}}$, Yugao Sun ${ }^{2, \mathrm{c}}$, Ang $\mathrm{Li}^{1, \mathrm{~d}}$, Yuying $\mathrm{Fu}^{1, \mathrm{f}}$ \\ ${ }^{1}$ College of Food Science and Biotechnology, Zhejiang Gongshang University, Xuezheng Road 18, \\ Hangzhou, China, 310018 \\ ${ }^{2}$ College of Chemistry and Materials Engineering, Wenzhou University, University Town, Chashan, \\ Wenzhou, China, 325035 \\ apanweichun1@yahoo.com, ’xujin@wzu.edu.cn, 'snygao@163.com, Iliang@mail.zjgsu.edu.cn, \\ ewebfu@163.com \\ *the corresponding author
}

Keywords: Crystal structure; Chiral symmetry breaking; Nucleation; Natural crystal growth

\begin{abstract}
The chiral symmetry breaking during the nucleation and growth of sodium chlorate and sodium bromate from their aqueous solutions was investigated under natural unstirred conditions. The results suggested that temperature fluctuation inherently existing in nature is a possible main driving force causing fluctuating supersaturation conditions, under which the chiral symmetry breaking is induced. Density gradient in the solution associated with water evaporation and solute concentration variation causes natural convection, which was found to amplify the existed biased handed structure to yield high value of the absolute crystal enantiomeric excess (CEE) in the absence of external stirring. Experimental results also showed that the chiral symmetry breaking process induced by temperature fluctuation is not significantly affected by the presence of achiral solid particles.
\end{abstract}

\section{Introduction}

Several profound research findings aiming at clarifying the mechanisms of cell-structure formation [1, 2], nucleotides synthesis [3] and RNA self-replication [4], have recently been reported in literatures. However, the achievement of homochirality of chiral molecules [5], which is another essential phenomenon pre-requested for the origin of life on our planet, remains a mystery. Kondepudi et al., in their seminal work [6], observed that stirring supersaturated sodium chlorate $\left(\mathrm{NaClO}_{3}\right)$ solutions resulted in crystals with high optical (enantiomorphic) purity while the crystals obtained in experiments without stirring under constant temperature were overall optically symmetric [7]. The crystallizations of $\mathrm{NaClO}_{3}$ and its close alternative, sodium bromate $\left(\mathrm{NaBrO}_{3}\right)$, from their aqueous solutions have since become widely used processes to emulate chiral evolution from symmetry to complete breaking [8,9]. The effects of fluidic condition has also been experimentally investigated [10]. On the theoretical side, pioneered by Frank's study on closed systems[11], several scenarios, such as "mother crystal" [12,13] and secondary nucleation amplification [6], have been proposed to explain chiral symmetry breaking. It should be noted that the above studies were focused on stirred solutions and under precisely controlled temperature, which are significantly different from the realistic conditions on primitive earth.

In this article, we studied a mechanism controlling chiral symmetry breaking under realistic conditions. The nucleation and growth of crystals in $\mathrm{NaClO}_{3}$ and $\mathrm{NaBrO}_{3}$ solutions were used as the model systems to monitor the evolution to homochirality without stirring and precise temperature control. The water evaporation caused crystal appearance in solutions. Since crystal has chiral property, the distribution of handed crystals was used to evaluate enantoiselective evolution. The results revealed that the initial mirror imbalance resulting from the intrinsic chiral fluctuations can be amplified by the prevalent daily temperature fluctuation. Further investigation showed that high supersaturation is favorable for the formation of pure handed crystals. 


\section{Materials and method}

Sodium bromate $\left(\mathrm{NaBrO}_{3}\right)$ crystallization without any control. $10 \mathrm{ml} \mathrm{NaBrO}_{3}$ (Waco, Japan) solution $\left(26.65 \%\right.$, weight, solute/solvent, the saturated concentration at $20^{\circ} \mathrm{C}$. [14]) was prepared at $40{ }^{\circ} \mathrm{C}$ and then transferred into a $100 \mathrm{ml}$ standard beaker, giving an initial liquid height of $0.5 \mathrm{~cm}$. The beaker was loosely covered by Parafilm (from Pechiney Plastic Packaging Company), which prevents the sample from being polluted by solid particles in the air while allows for free water evaporation. The whole system was then placed on a laboratory desk without any stirring and temperature control for three days. To our knowledge, no studies using such an uncontrolled system have been reported in the literatures. Temperature fluctuation in the laboratory was measured to be in the range of $15-23{ }^{\circ} \mathrm{C}$ during these days. The chiral purity of obtained crystals was evaluated by the crystal enantiomeric excess (CEE) defined as [15]:

$$
\mathrm{CEE}=\frac{\mathrm{N}_{\mathrm{L}}-\mathrm{N}_{\mathrm{D}}}{\mathrm{N}_{\mathrm{L}}+\mathrm{N}_{\mathrm{D}}}
$$

where $\mathrm{N}_{\mathrm{L}}$ and $\mathrm{N}_{\mathrm{D}}$ are numbers of L- and D- crystals, respectively.

Since the size of crystals obtained in our experiments was large enough $(>1 \mathrm{~mm})$, the method proposed by Kondepudi [6] was employed to determine CEE. With the aid of white light source, a pair of polarizers has been used to distinguish and count L- and D- crystals. Pictures of crystals in the solution were taken using an Olmpus E-1 digital camera equipped with a $35 \mathrm{~mm}$ F3.5 macro lens. Representative results are shown in Fig. 1.

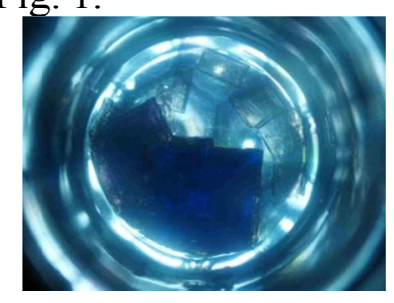

Figure 1. The chirality of the $\mathrm{NaClO}_{3}$ crystals was determined by rotating one of the crossed polarizers. As a result, the $\mathrm{L}$ crystals become dark.

$\mathrm{NaBrO}_{3} / \mathrm{NaClO}_{3}$ crystallization: different solution heights. Two parallel experiments of $\mathrm{NaBrO}_{3}$ crystallization were carried out in two identical beakers $(6 \mathrm{~cm}$ diameter $)$ with the same solute concentration as "Sodium bromate $\left(\mathrm{NaBrO}_{3}\right)$ crystallization without any control". The only difference between them was the initial liquid height, one being $0.5 \mathrm{~cm}$ and the other one being 3.5 $\mathrm{cm}$. Over three days, crystals in solutions were separated, identified for handed structure and counted.

For generality, a similar pare of operations were carried out using $\mathrm{NaClO}_{3}$. $180.9 \mathrm{~g} \mathrm{NaClO}$ (Aladdin, China, CP) was dissolved in $150 \mathrm{ml}$ purified water at $50{ }^{\circ} \mathrm{C}$.

$\mathrm{NaClO}_{3}$ crystallization: temperature fluctuation range. Three parallel experiments were carried out to evaluate the influence of the range of temperature fluctuation. In each experiment, $60.3 \mathrm{~g} \mathrm{NaClO}_{3}$ was added to $50 \mathrm{ml}$ purified water in a $250 \mathrm{ml}$ beaker separately immersed in a water bath initially controlled at $50{ }^{\circ} \mathrm{C}$. Parafilm was used to isolate the solution from atmosphere and prevent water evaporation, forming a close system. After all powders were completely dissolved and temperature was equilibrated, three water baths (Julabo F34, Circulator) were simultaneously reset to $35{ }^{\circ} \mathrm{C}, 30{ }^{\circ} \mathrm{C}$ and $25{ }^{\circ} \mathrm{C}$, respectively, with the cooling function switched off. Over three days, CEE of the obtained crystals was monitored.

$\mathrm{NaClO}_{3}$ crystallization: free surface area of water evaporation. $\mathrm{NaClO}_{3}$ solution with same solute concentration as " $\mathrm{NaBrO}_{3} / \mathrm{NaClO}_{3}$ crystallization: different solution heights" was been prepared at $45{ }^{\circ} \mathrm{C}$ and then this solution distributed to 4 small $100 \mathrm{ml}$ beakers $(6 \mathrm{~cm}$ diameter) and 4 $500 \mathrm{ml}$ large beakers $\left(8 \mathrm{~cm}\right.$ diameter) kept at the same temperature in order to avoid $\mathrm{NaClO}_{3}$ crystallization during this process. The heights of solution in all beakers were same at $3.5 \mathrm{~cm}$. All beakers were then put in water baths set at $40{ }^{\circ} \mathrm{C}$. Over one day, crystalls in all beakers were monitored. 
$\mathrm{NaClO}_{3}$ crystallization: the presence of solid particles without chirality. A same operation as "Sodium bromate $\left(\mathrm{NaBrO}_{3}\right)$ crystallization without any control" was repeated with the addition of $10 \mathrm{mg}$ silicon dioxide beads ( $30 \mathrm{~m}$ diameter, Mitsubishi Material) to the $\mathrm{NaBrO}_{3}$ solution sample.

$\mathrm{NaClO}_{3}$ crystallization: solvable impurity.Experiment "Sodium bromate $\left(\mathrm{NaBrO}_{3}\right)$ crystallization without any control" was repeated using reagent grade chemicals (purity greater than $99 \%$, Waco, Japan $)$ and at a lower temperature $\left(20{ }^{\circ} \mathrm{C}\right)$ for the initial dissolving process.

All the above mentioned experiments were repeated for at least 4 times.

\section{Results and Discussions}

High chiral purity obtained at uncontrolled conditions. In our experiment, there existed not only nucleation of crystal but also crystal growth. Therefore, we need to investigate both factors.

The absolute CEE (|CEE|) of obtained crystals in "Sodium bromate $\left(\mathrm{NaBrO}_{3}\right)$ crystallization without any control" experiment was determined to be $96 \%$. As a comparison, a rather low absolute CEE value less than $1 \%$ was obtained in a parallel experiment with a constant temperature controlled at $22 \pm 1{ }^{\circ} \mathrm{C}$, which is a normal operation in chiral separation observation in $\mathrm{NaClO}_{3}$ starting as early as Kipping and Pope[16]. These observations cannot be explained by the mechanism of natural convection [17], which attributes chiral crystal proliferation to the secondary nucleation companying with the hydrodynamic motion, because the achievement of high CEE requires the existence of a dominative chiral structure nuclei in homogeneous nucleation processes. Viedma [18] showed that high supersaturation causes almost complete chiral symmetry breaking. And an elegant observation in boiling solution system elaborates that there exists chiral cluster with CEE dependency of supersaturation before primary nucleation[19]. Furthermore, our observations indicated that temperature fluctuation intrinsically existing in nature may cause chiral symmetry breaking which is initiated by chiral fluctuation [20]. Taking both into account, we hypothesize that high supersaturation caused by temperature fluctuation in nature is a possible main driving force for chiral symmetry breaking. In order to verify this hypothesis, three sets of experiments were performed.

The influence of solution volume. The average absolute CEE values for the 0.5 and $3.5 \mathrm{~cm}$ initial heights were measure to be $64 \%$ and $16 \%$ (Table1), respectively. Since the two beakers experienced similar temperature fluctuation and have identical surface areas, it is reasonable to assume that overall evaporation rates were close in the two experiments (neglecting solute concentration effects). Therefore, the sample with smaller volume had a relatively larger supersaturation rate and, as expected, gave a higher absolute CEE value. Similar experiments were also performed using $\mathrm{NaClO}_{3}$ for more generality and similar results were obtained (see Table 1).

Table 1 the influence of solution volume

\begin{tabular}{|c|c|c|c|c|c|}
\hline No. & Solute & Initial concentration (w \%) & Height & Average CEE (\%) & SD (\%) \\
\hline 1 & $\mathrm{NaBrO}_{3}$ & 26.65 & $0.5 \mathrm{~cm}$ & 64 & 31 \\
\hline 2 & $\mathrm{NaBrO}_{3}$ & 26.65 & $3.5 \mathrm{~cm}$ & 16 & 11 \\
\hline 3 & $\mathrm{NaClO}_{3}$ & 49.3 & $0.5 \mathrm{~cm}$ & 72 & 37 \\
\hline 4 & $\mathrm{NaClO}_{3}$ & 49.3 & $3.5 \mathrm{~cm}$ & 20 & 13 \\
\hline $1 \mathrm{a}^{*}$ & $\mathrm{NaBrO}_{3}$ & 26.65 & $0.5 \mathrm{~cm}$ & 62 & 28 \\
\hline $1 \mathrm{~b}^{* *}$ & $\mathrm{NaBrO}_{3}$ & 26.65 & $0.5 \mathrm{~cm}$ & 68 & 37 \\
\hline
\end{tabular}

*: repeating No. 1 with beads

**: repeating No. 1 with impurities eliminated

The influence of temperature fluctuation range. Motivated by the temperature fluctuation observation of Noorduin et al.[21], the second set consists of three parallel experiments that have been described in section " $\mathrm{NaClO}_{3}$ crystallization: temperature fluctuation range". As shown in Table 2, high absolute CEE values were obtained for all three experiments. Since the solubility of $\mathrm{NaClO}_{3}$ is sensitive to temperature, high saturation rates during these highly temperature descending processes were expected. While complete chiral symmetry breaking were observed in $35{ }^{\circ} \mathrm{C}$ and $30^{\circ} \mathrm{C}$ experiments, the average absolute $\mathrm{CEE}$ value of $25^{\circ} \mathrm{C}$ experiments, which had the 
highest supersaturation rate, was found to be reduced from $100 \%$ to $90 \%$. This may be attributed to serious aggregation at a too high supersaturation rate.

Table 2 the influence of temperature fluctuation range

\begin{tabular}{|c|c|c|c|c|}
\hline No. & Cooling temperature $\left({ }^{\circ} \mathrm{C}\right)$ & $\mathrm{N}_{\mathrm{L}}$ & $\mathrm{N}_{\mathrm{R}}$ & $\mathrm{ECC}(\%)$ \\
\hline 1 & 35 & 209 & 0 & 100 \\
\hline 2 & 35 & 315 & 0 & 100 \\
\hline 3 & 35 & 189 & 0 & 100 \\
\hline 4 & 35 & 0 & 214 & -100 \\
\hline 5 & 30 & 324 & 0 & 100 \\
\hline 6 & 30 & 0 & 228 & -100 \\
\hline 7 & 30 & 0 & 278 & -100 \\
\hline 8 & 30 & 156 & 0 & 100 \\
\hline 9 & 25 & 321 & 28 & 84 \\
\hline 10 & 25 & 248 & 5 & 96 \\
\hline 11 & 25 & 12 & 286 & -92 \\
\hline 12 & 25 & 24 & 380 & -88 \\
\hline
\end{tabular}

The influence of free water evaporation area. As shown in Table 3, the smaller free water evaporation area caused higher absolute CEE. Water evaporation caused local temperature, composition and density gradients. Usually the fraction of solution close to evaporation surface has higher density, resulting in natural convection in the system [22]. The velocity associated with this natural convection can be estimated by [23]

$$
\mathrm{u}=\frac{\mathrm{g} \Delta \rho \mathrm{L}^{2}}{\mu}
$$

where $\mathrm{u}$ is the velocity in solution, $\mathrm{g}$ is the acceleration of gravity, $\mathrm{L}$ is the height of solution and $\mu$ is the solution viscosity. Since all solutions have the same thermal and mechanic experience, it is reasonable to assume that $\Delta \rho$ is same in all experiments. In addition, difference among the solutions in viscosity can also be neglected. The convection velocity was therefore similar in all experiments. However, the non-slip boundary condition requires fluidic velocity equal to zero at the container wall [24]. Therefore, there exists a velocity gradient along the radius direction, resulting a shear force that can be roughly estimated by

$$
\tau=\frac{\mathrm{u}-0}{\mathrm{r}} \mathrm{u}=\mathrm{g} \Delta \rho \mathrm{L}^{2} / \mu
$$

$\tau=\frac{\mathrm{u}-0}{\mathrm{r}}$ where $\mathrm{r}$ is the radius of the container. Under the action of this shear force, the whiskers formed at the evaporation surface can be broken into smaller ones during their downward movement towards the beaker bottom, resulting in an increased number of crystal seeds with the same chirality. Cartwright reported that the fluid movement is one source for handed structure amplification in $\mathrm{NaClO}_{3}$ solutions [25]. According to Eq. 3, larger shear force exists in the smaller beakers, resulting in higher absolute CEE of the obtained crystals as shown in Table 3 . It should be mentioned that, during the experiments under temperature control, in the absence of nature convection, the constant stirring played the role of providing local fluid movement and the shear force needed for chirality amplification.

Table 3 the influence of free water evaporation area

\begin{tabular}{|c|c|c|c|c|}
\hline No. & Surface diameter $(\mathrm{cm})$ & $\mathrm{N}_{\mathrm{L}}$ & $\mathrm{N}_{\mathrm{R}}$ & ECC (\%) \\
\hline 1 & 6 & 10 & 249 & -92 \\
\hline 2 & 6 & 266 & 10 & 93 \\
\hline 3 & 6 & 8 & 276 & -94 \\
\hline 4 & 6 & 283 & 28 & 82 \\
\hline 5 & 8 & 124 & 816 & -74 \\
\hline 6 & 8 & 892 & 622 & 18 \\
\hline 7 & 8 & 87 & 822 & -81 \\
\hline 8 & 8 & 428 & 882 & -35 \\
\hline
\end{tabular}


The influence of achiral solvable and dissolvable impurity.In the above discussions, we proposed and verified that chiral symmetry breaking can be induced by free temperature fluctuation under unstirred conditions. The inherent convection amplifies already biased handed structure. Compared with previous studies, the conditions of above experiments are closer to nature environments. However, nature conditions can by no means be completely duplicated in the laboratory. An inherently logical question is whether other variables besides temperature have significant effects on chiral symmetry breaking. In this study, experiments were carried out to investigate the effects of solid particles and solvable impurities. The results are discussed in this section.

The existence of solid particle in solution is almost inevitable in nature. Many efforts have been made to investigate the effects of particles with chiral surface characteristics $[26,27]$. Since this study is mainly focused on the origin of chirality, particles without chirality are more relevant. In this case, the concerned issue is the possible supersaturation rate reduction resulting from heterogeneous nucleation [28]. Intrigued by Qian and Botsaris's observation on artificial seeds in stirred $\mathrm{NaClO}_{3}$ solutions [28], experiment described in section " $\mathrm{NaClO}_{3}$ crystallization: the presence of solid particles without chirality" was performed. The CEE value of obtained crystals is compared with the original one in Table 1. It may be seen that there is no significant difference between the results obtained with and without silicon beads. This indicates that the heterogeneous nucleation induced by silicon particles, the most common solid particles existing on primitive earth, has little influence on chiral symmetry breaking.

\section{Conclusion}

Chiral symmetry breaking during the nucleation and growth of $\mathrm{NaClO}_{3}$ and $\mathrm{NaBrO}_{3}$ from their aqueous solutions was investigated under the conditions without stirring and precise temperature control. Natural temperature fluctuation was found to be a possible driving force to build up the high supersaturation rate and, hence, induce the remarkable phenomena of chiral symmetry breaking. It was also observed that the chiral purity of obtained crystals is slightly reduced under too high temperature fluctuation, which may be attributed to the serious aggregation occurring at a too high supersaturation rate. A density gradient in the solution is formed with water evaporation and thereafter generates a natural convection. The shear force associated with this convection amplifies the existed biased handed structure to the yield high absolute CEE value of obtained crystals. In addition to the relaxed conditions in term of no constant stirring, an important feature of the chiral symmetry breaking induced by temperature fluctuation is its robustness. Normally existing solid particles barely have any influence on the CEE value of obtained crystals. Remarkably, considering the fact that chirality formation is a fundamental phenomena pre-requested for the origin of life, our observations partially coincides with Darwin's theory [29] that "the origin of life started in the pond which endured cycles of rain and evaporation".

\section{Acknowledgements}

This work is supported by grants from the National Natural Science Foundation of China (31171713, 21106108), the program of Zhejiang Education Department (Y201119393) and the Initial Funding of Zhejiang Gongshang University to W. Pan.

\section{Reference}

[1] S.S. Mansy, J.P. Schrum, M. Krishnamurthy, S. Tobé, D.A. Treco, J.W. Szostak, Template-directed Synthesis of a Genetic Polymer in a Midel Protocell, Nature, 454 (2008) 122-125.

[2] J.W. Szostak, D.P. Bartel, P.L. Luisi, Synthesizing Life, Nature, 410 (2001) 715-718. 
[3] M.W. Powner, B. Gerland, J.D. Sutherland, Synthesis of Activated Pyrimidine Ribonucleotides in Prebiotically Plausible Conditions, Nature, 459 (2009) 239-242.

[4] T.A. Lincoln, G.F. Joyce, Self-Sustained Replication of an RNA Enzyme, Science, (2009) 1229-1232.

[5] W.L. Noorduin, T. Izumi, A. Millemaggi, M. Leeman, H. Meekes, W.J.P.V. Enckevort, R.M. Kellogg, B. Kaptein, E. Vlieg, D.G. Blackmond, Emergence of a Single Solid State from a Nearly Racemic Amino Acid Derivative, Journal of the American Chemical Society, 130 (2008) 1158-1159.

[6] D.K. Kondepudi, R.J. Kaufman, N. Singh, Chiral Symmetry Breaking in Sodium Chlorate Crystallization, Science, 250 (1990) 975-976.

[7] F.S. Kipping, W.J. Pope, LXIII.-Enantiomorphism, Journal of the Chemical Society, Transactions, 73 (1898) 606-617.

[8] G. Wald, The Origin of Optical Activity, Annals of the New York Academy of Sciences, 69 (1957) 352-368.

[9] I.R. Epstein, The consequences of imperfect mixing in autocatalytic chemical and biological systems, Nature, 374 (1995) 321-327.

[10] A. Brandenburg, T. Multamāki, How long can left and right handed life forms coexist?, International Journal of Astrobiology, 3 (2004) 209-219.

[11] F.C. Frank, On Spontaneous Asymmetric Synthesis, Biochimica et Biophysica Acta, 11 (1953) 459-463.

[12] D.K. Kondepudi, K.L. Bullock, J.A. Digits, P.D. Yarborough, Stirring Rate as a Critical Parameter in Chiral Symmetry Breaking Crystallization, Journal of the American Chemical Society, 117 (1995) 401-404.

[13] T. Buhse, D. Durand, D. Kondepudi, J. Laudadio, S. Spiker, Chiral Symmetry Breaking in Crystallization: The Role of Convection, Phys. Rev. Lett., 84 (2000) 4405-4408.

[14] Handbook of Chemistry and Physics, 81-th ed., CRC, Boca Raton, FL.

[15] C. Viedma, Chiral Symmetry Breaking During Crystallization: Complete Chiral Purity Induced by Nonlinear Autocatalysis and Recycling, Phys. Rev. Lett., 94 (2005) 065504.

[16] F.S. Kipping, W.J. Pope, Enantiomorphism, Journal of the Chemical Society, Transactions 73 (1898) 606-617.

[17] M. Szurgot, J. Szurgot, Chiral Symmetry Breaking in Sodium Chlorate Crystallization from Unstirred Solution, Crystal Research and Technology 30 (1995) 949-956.

[18] C. Viedma, Experimental Evidence of Chiral Symmetry Breaking in Crystallization from Primary Nucleation, J. Cryst. Growth, 261 (2004) 118-121.

[19] Z. El-Hachemi, J. Crusats, J.M. Ribó, J.M. McBride, S. Veintemillas-Verdaguer, Metastability in Supersaturated Solution and Transition towards Chirality in the Crystallization of $\mathrm{NaClO}_{3}$, Angewandte Chemie International Edition, 50 (2011) 2359-2363.

[20] K. Mislow, Absolute Asymmetric Synthesis: A Commentary, Collection of Czechoslovak Chemical Communications, 68 (2003) 849-864.

[21] W.L. Noorduin, H. Meekes, A.A.C. Bode, W.J.P.v. Enckevort, B. Kaptein, R.M. Kellogg, E. Vlieg, Explanation for the Emergence of a Single Chiral Solid State during Attrition - Enhanced Ostwald Ripening: Survival of the Fittest, Crystal Growth and Design, 8 (2008) 1675-1681. 
[22] C.O. Bennett, J.E. Myers, Momentum, heat and mass transfer, McGraw_Hill, New York, 1962.

[23] W.M. Kays, M.L. Crawford, B. Weigand, Convective Heat and Mass Transfer, McGraw-Hill Science/Engineering/Math, New York, 2005.

[24] P.K. Kundu, I.M. Cohen, Fluid Mechanics, Academic Press, London, 2002.

[25] J.H.E. Cartwright, J.M. García-Ruiz, O. Piro, C.I. Sainz-Díaz, I. Tuval, Chiral Symmetry Breaking during Crystallization: An Advection-Mediated Nonlinear Autocatalytic Process, Phys. Rev. Lett., 93 (2004) 035502.

[26] W.A. Bonner, P.R. Kavasmaneck, F.S. Martin, J.J. Flores, Asymmetric Adsorption by Quartz: a Model for the Prebiotic Origin of Optical Activity Origins of Life and Evolution of Biosphere, 6 (1975) 367-376.

[27] R.M. Hazen, T.R. Filley, G.A. Goodfriend, Selective adsorption of L- and D- amino acids on calcite: Implications for biochemical homochirality, Proc. Natl. Acad. Sci. U. S. A., 98 (2001) 5487-5490.

[28] R.-Y. Qian, G.D. Botsaris, Nuclei Breeding from a Chiral Crystal Seed of $\mathrm{NaBrO}_{3}$, Chemical Engineering Science, 53 (1998) 1745-1756.

[29] C. Wills, J. Bada, The Spark of Life: Darwin and the Primeval Soup, Perseus, Cambridge,MA, 2000. 